\title{
DISTRIBUIÇÃO E ABUNDÂNCIA DE PEIXES BENTÔNICOS EXPLOTADOS PELOS LINHEIROS AO LARGO DO SUDESTE DO BRASIL (1986-1995)
}

\author{
PAIVA, M. P. e ANDRADE-TUBINO, M. F. \\ Departamento de Biologia Marinha, Universidade Federal do Rio de Janeiro, \\ Cidade Universitária, Ilha do Fundão, CEP 21944-970, Rio de Janeiro, RJ \\ Correspondência para: Melquíades Pinto Paiva, Departamento de Biologia Marinha, Universidade \\ Federal do Rio de Janeiro, Cidade Universitária, Ilha do Fundão, CEP 21944-970, Rio de Janeiro, RJ \\ Recebido em 17/10/97 - Aceito em 13/07/98 - Distribuído em 30/11/98
}

\section{ABSTRACT \\ Distribution and abundance of benthonic fishes exploited by liners off southeast Brazil (1986 - 1995)}

This paper deals with the distribution and abundance of benthonic fishes main species exploited by liners off southeast Brazil (latitudes $18^{\circ}-26^{\circ}$ S), in the period 1986-1995, listed as follows, with their respective participations in the whole catches: tilefish $=$ Lopholatilus vilariii Ribeiro $=33.4 \%$, sandperch $=$ Pseudopercis numida Ribeiro $=18.4 \%$, snowy grouper $=$ Epinephelus niveatus $($ Valenciennes $)=12.1 \%$ grouper, $=$ Epinephelus marginatus $($ Lowe $)=4.1 \%$, and black grouper $=$ Mycteroperca bonaci $($ Poey $)$ $=3.1 \%$. The fisheries were conducted in tropical (latitudes $18^{\circ}-23^{\circ} \mathrm{S}$ ) and subtropical (latitudes $23^{\circ}-$ $26^{\circ} \mathrm{S}$ ) waters, having the Cabo Frio upwelling as zoogeographical divisor. The data were grouped by latitude and depth bands, according to species and year seasons.

The fisheries were greatly concentrated in subtropical waters, with decreasing productivity trend(s) as increase(s) latitude and/or depth of exploited fishing grounds. Grouper and black grouper were the dominant species in tropical waters, clearly between latitudes $18^{\circ}-20^{\circ} \mathrm{S}$, with major catches until 80 meters in depth. Tilefish, sandperch, and snowy grouper always were dominant species in subtropical waters, with major catches made in depths higher than 100 meters, including the upper continental slope. The main five species showed seasonal variation trends in production, but only the snowy grouper seems to carry out seasonal displacement along the coast. The lower diversity and higher biomass of benthonic fishes were confirmed in subtropical waters.

Key words: benthonic fishes, distribution and abundance, liner's fisheries, southeast Brazil.

\section{RESUMO}

Este trabalho trata da distribuição e abundância das principais espécies de peixes bentônicos explotados pelos linheiros ao largo do sudeste do Brasil (latitudes $18^{\circ}-26^{\circ} \mathrm{S}$ ), no período 1986-1995, listadas a seguir, com as respectivas participações no total das capturas: batata $=$ Lopholatilus vilariii Ribeiro $=33,4 \%$, namorado $=$ Pseudopercis numida Ribeiro $=18,4 \%$, cherne $=$ Epinephelus niveatus $($ Valenciennes $)=12,1 \%$, garoupa $=$ Epinephelus marginatus $($ Lowe $)=4,1 \%$ e badejo $=$ Mycteroperca bonaci $($ Poey $)=3,1 \%$. As pescarias ocorreram em águas tropicais (latitudes $18^{\circ}-23^{\circ} \mathrm{S}$ ) e subtropicais (latitudes $23^{\circ}-26^{\circ} \mathrm{S}$ ), tendo como divisor zoogeográfico a ressurgência de Cabo Frio.

Os dados foram agrupados por faixas de latitude e de profundidade, segundo espécies e estações do ano. As pescarias estiveram grandemente concentradas nas águas subtropicais, com tendência(s) de produtividade decrescente, à medida que aumenta $(\mathrm{m})$ a latitude e/ou a profundidade dos pesqueiros explotados. Nas águas tropicais, as espécies dominantes foram a garoupa e o badejo, de forma indiscutível entre as latitudes $18^{\circ}-20^{\circ} \mathrm{S}$, com maiores capturas em profundidades de até 80 metros. Nas águas subtropicais, as espécies dominantes sempre foram o batata, o namorado e o cherne, com maiores 
capturas em profundidades superiores a 100 metros, incluindo a parte superior do talude continental. A produção das cinco espécies principais apresentou tendências de variação estacional, mas apenas o cherne evidenciou deslocamento sazonal ao longo da costa. Foram comprovadas a menor diversidade e a maior biomassa de peixes bentônicos nas águas subtropicais.

Palavras-chave: peixes bentônicos, distribuição e abundância, pescarias de linheiros, sudeste do Brasil.

\section{INTRODUÇÃO}

As pescarias de barcos linheiros, ao largo da costa sudeste do Brasil, capturam peixes de grande importância econômica, entre os quais se destacam as seguintes espécies, na ordem decrescente dos volumes desembarcados nos anos de 1986-1995: batata (tilefish) $=$ Lopholatilus vilariii Ribeiro - Branchiostegidae, com 33,4\% dos desembarques; namorado (sandperch) $=$ Pseudopercis $n u$ mida Ribeiro - Pinguipedidae, com 18,4\% dos desembarques; cherne (snowy grouper) = Epinephelus niveatus (Valenciennes) - Serranidae, com $12,1 \%$ dos desembarques; garoupa (grouper) = Epinephelus marginatus (Lowe) - Serranidae, com 4,1\% dos desembarques; badejo (black grouper $)=$ Mycteroperca bonaci $($ Poey $)-$ Serranidae, com $3,1 \%$ dos desembarques. Em conjunto, estas cinco espécies alcançaram o montante de $71,1 \%$ dos desembarques totais da frota de linheiros, na região e período considerados.

Em trabalhos anteriores, estudamos, as referidas pescarias, conforme indicamos, especificando áreas e períodos: áreas de Abrolhos e Mar Novo (sudeste do Brasil), consideradas em conjunto, com dados de 1979-1985 (Paiva \& Andrade, 1994); apenas o sudeste do Brasil, com dados de 19791995 (Paiva \& Andrade-Tubino, 1998).

Agora, vamos cuidar somente da distribuição e abundância das espécies acima mencionadas, ao largo da costa sudeste do Brasil (latitudes $18^{\circ}-26^{\circ} \mathrm{S}$ ), por faixas de latitude e profundidade, considerando as diferentes estações (trimestres), com base nos dados de pescarias realizadas em águas tropicais (latitudes $18^{\circ}-23^{\circ} \mathrm{S}$ ) e águas subtropicais (latitudes $23^{\circ}-26^{\circ} \mathrm{S}$ ), nos anos de 1986-1995 .

De certa forma, este trabalho complementa dois outros, anteriormente publicados, a respeito das mesmas espécies, com dados de 19861989, sem levar em conta a distribuição e a abundância dos peixes por faixas de profundidade (Andrade, 1995; Paiva, 1997).

\section{MATERIAL E MÉTODOS}

Os dados utilizados neste trabalho são oriundos dos mapas de bordo da frota de linheiros baseados nos portos do Rio de Janeiro/Niterói (Estado do Rio de Janeiro) e de Vitória (Estado do Espírito Santo), correspondentes a desembarques efetuados nos anos de 1986-1995, controlados pelo Instituto Brasileiro do Meio Ambiente e dos Recursos Naturais Renováveis - IBAMA (Tabelas 1 e 2).

Foram consideradas apenas as pescarias efetuadas ao largo da costa sudeste do Brasil, compreendendo os Estados do Espírito Santo, Rio de Janeiro e São Paulo, nos anos de 1986-1995, entre as latitudes $18^{\circ}-26^{\circ} \mathrm{S}$.

Os dados das pescarias foram processados segundo as seguintes variáveis, considerando o total das capturas e aquelas correspondentes a cada uma das espécies destacadas: trimestres (estações), produção e esforço de pesca, faixas de latitude e de profundidade, águas tropicais e subtropicais, composição da biocenose e abundância relativa.

O divisor entre as águas tropicais e subtropicais está na altura da ressurgência de Cabo Frio (Silva, 1973; Valentin, 1984), muito evidente no tocante à ictiofauna (Briggs, 1974; Moyle \& Cech Jr., 1982; Menni, 1983)

A metodologia de análise adotada é bastante simples, procurando mostrar as variações estacionais (trimestres) e espaciais (latitudes e profundidades), considerando as capturas de cada uma das espécies estudadas.

\section{SÚMULAS BIOLÓGICAS}

As fontes bibliográficas que dão suporte às informações apresentadas nesta parte do trabalho são as seguintes: Ihering (1940), 1968; Santos (1949), 1982; Figueiredo \& Menezes, 1980; Nagelkerken, 1981; Suzuki (1983), 1993; Nomura, 1984; Menezes \& Figueiredo, 1985; Szpilman, 1991; Carvalho Filho, 1992; Heemstra \& Randall, 1993; 
Vazzoler, 1993; Andrade, 1995; Paiva \& FontelesFilho, 1995; Paiva, 1997; Machado et alii, 1997.

Badejo - Encontrado em águas tropicais e subtropicais do Oceano Atlântico; na sua parte ocidental, desde Massachusetts (Estados Unidos da América) e Bermudas até São Paulo (Brasil). Em nosso país, é mais comum em águas tropicais, nas regiões nordeste e sudeste. Habitante de fundos rochosos e coralinos com até 150 metros de fundura, mais comumente entre 30 e 60 metros de profundidade; apenas os jovens vivem próximos da costa, porém os adultos se afastam muito dela. Preferencialmente, alimenta-se de crustáceos e peixes bentônicos.

Batata - Encontrado em águas tropicais, subtropicais e temperadas do Oceano Atlântico, desde o Arquipélago dos Abrolhos (Brasil) até o norte da Argentina. Habitante de fundos arenosos e rochosos, normalmente além de 50 metros de profundidade, nas proximidades de parcéis, montes submersos e ilhas oceânicas. Alimenta-se de invertebrados e peixes bentônicos.

Cherne - Ocorre nos Oceanos Atlântico e Pacífico. No Atlântico Ocidental é encontrado desde Massachusetts (Estados Unidos da América) até o Rio Grande do Sul (Brasil). Em nosso país, é mais comum nas regiões nordeste e sudeste. Habitante de fundos lodosos, arenosos e rochosos: os jovens preferem águas rasas, nos estuários e proximidades de costões rochosos e de recifes; os adultos vivem em águas entre 30 e 500 metros de profundidade.
Garoupa - Ocorre nos dois lados do Oceano Atlântico e no Mar Mediterrâneo. No Atlântico Ocidental é encontrada desde o nordeste do Brasil até a Argentina. Prefere fundos rochosos e coralinos, entre parcéis e em profundidades de até 80 metros. Alimenta-se de cefalópodos, crustáceos e peixes bentônicos.

Namorado - Tem ocorrência restrita ao litoral brasileiro, desde o Espírito Santo até o Rio Grande do Sul (Haimovici et al., 1996). Prefere os fundos de areia, cascalho e rocha, entre 50 e 100 metros de profundidade, com maior ocorrência em parcéis profundos e ao redor de ilhas oceânicas. Alimenta-se de crustáceos e peixes bentônicos.

\section{RESULTADOS E DISCUSSÃO}

As pescarias da frota de linheiros, ao largo da costa sudeste do Brasil, são grandemente concentradas em suas águas subtropicais, com tendência(s) de produtividade decrescente à medida que aumenta(m) a latitude e/ou a profundidade dos pesqueiros explotados (Tabelas 1 e 2).

Em geral, as espécies estudadas preferem águas tropicais ou subtropicais, bem como faixas de profundidade, o que é bem evidente na distribuição de suas capturas pela frota de linheiros (Tabelas 3 e 4).

Tomando-se a produção pesqueira como representativa da biomassa dos peixes bentônicos, ao largo da costa sudeste do Brasil, os dados disponíveis (Tabela 3) possibilitam os seguintes destaques:

TABELA 1

Produção total de pescado, esforço de pesca e abundância (CPUE = kg/anzol/dia) de peixes bentônicos, capturados por barcos linheiros ao largo da costa sudeste do Brasil, por faixas de latitude, no período 1986-1995.

\begin{tabular}{|c|c|c|c|c|c|}
\hline \multirow{2}{*}{$\begin{array}{c}\text { Latitudes } \\
\left({ }^{\circ} \mathbf{S}\right)\end{array}$} & \multicolumn{2}{|c|}{ Produção } & \multicolumn{2}{|c|}{ Anzóis/dia } & \multirow{2}{*}{$\begin{array}{c}\text { CPUE } \\
(\mathbf{k g})\end{array}$} \\
\hline & kg & $\%$ & $\mathbf{n}^{0}$ & $\%$ & \\
\hline 18-19 & 1.467 .200 & 9,0 & 696.869 & 3,7 & 2,1 \\
\hline $19-20$ & 247.046 & 1,5 & 138.347 & 0,7 & 1,8 \\
\hline $20-21$ & 102.860 & 0,6 & 88.064 & 0,5 & 1,2 \\
\hline 21-22 & 1.710 .807 & 10,4 & 733.005 & 3,9 & 2,3 \\
\hline $22-23$ & 203.784 & 1,2 & 206.038 & 1,1 & 1,0 \\
\hline 23-24 & 6.874 .508 & 42,0 & 9.447 .942 & 50,4 & 0,7 \\
\hline 24-25 & 5.172 .553 & 31,6 & 6.449 .878 & 34,4 & 0,8 \\
\hline $25-26$ & 597.559 & 3,7 & 987.318 & 5,3 & 0,6 \\
\hline Totais & 16.376 .317 & 100,0 & 18.747 .461 & 100,0 & 0,9 \\
\hline
\end{tabular}


TABELA 2

Produção total de pescado, esforço de pesca e abundância (CPUE = kg/anzol/dia) de peixes bentônicos, capturados por barcos linheiros em águas tropicais $\left(18^{\circ}-23^{\circ} \mathrm{S}\right)$ e subtropicais $\left(23^{\circ}-26^{\circ} \mathrm{S}\right)$ ao largo da costa sudeste do Brasil, por faixas de profundidade, no período 1986-1995.

\begin{tabular}{|c|c|c|c|c|c|}
\hline \multirow{2}{*}{$\begin{array}{l}\text { Profundidades } \\
\text { (m) }\end{array}$} & \multicolumn{2}{|c|}{ Produção } & \multicolumn{2}{|c|}{ Anzóis/dia } & \multirow{2}{*}{$\begin{array}{c}\text { CPUE } \\
(\mathrm{kg})\end{array}$} \\
\hline & kg & $\%$ & n⿳- & $\%$ & \\
\hline \multicolumn{6}{|c|}{ * Águas tropicais $\left(18^{\circ}-23^{\circ} \mathrm{S}\right)$} \\
\hline--10 & 9.032 & 0,3 & 706 & 0,0 & 12,8 \\
\hline 11-20 & 111.119 & 3,6 & 16.224 & 1,1 & 6,8 \\
\hline 21-30 & 56.830 & 1,9 & 15.224 & 1,0 & 3,7 \\
\hline $31-40$ & 195.335 & 6,4 & 88.199 & 5,8 & 2,2 \\
\hline 41-50 & 461.652 & 15,1 & 195.730 & 12,8 & 2,4 \\
\hline $51-60$ & 852.205 & 28,0 & 468.331 & 30,5 & 1,8 \\
\hline 61-70 & 148.801 & 4,9 & 67.788 & 4,4 & 2,2 \\
\hline 71-80 & 42.415 & 1,4 & 13.440 & 0,9 & 3,2 \\
\hline $81-90$ & 4.367 & 0,1 & 2.160 & 0,1 & 2,0 \\
\hline 91-100 & 24.308 & 0,8 & 10.648 & 0,7 & 2,3 \\
\hline 101-110 & 80.754 & 2,6 & 27.125 & 1,8 & 3,0 \\
\hline 111-120 & 254.528 & 8,3 & 92.560 & 6,0 & 2,7 \\
\hline 121-130 & 264.732 & 8,7 & 124.170 & 8,1 & 2,1 \\
\hline 131-140 & 68.748 & 2,3 & 97.643 & 6,4 & 0,7 \\
\hline 141-150 & 9.306 & 0,3 & 17.677 & 1,2 & 0,5 \\
\hline $151-160$ & 166.351 & 5,5 & 65.772 & 4,3 & 2,5 \\
\hline 161-170 & 4.505 & 0,1 & 7.440 & 0,5 & 0,6 \\
\hline 171-180 & 124.193 & 4,1 & 62.647 & 4,1 & 2,0 \\
\hline 181-190 & 38.757 & 1,3 & 16.180 & 1,0 & 2,4 \\
\hline 191-200 & 130.483 & 4,3 & 143.358 & 9,3 & 0,9 \\
\hline 201- - & - & - & - & - & - \\
\hline Totais & 3.048 .421 & 100,0 & 1.533 .022 & 100,0 & 2,0 \\
\hline \multicolumn{6}{|c|}{ * Águas subtropicais $\left(23^{\circ}-26^{\circ} \mathrm{S}\right)$} \\
\hline--10 & 1.878 & 0,0 & 170 & 0,0 & 11,0 \\
\hline 11-20 & 37.784 & 0,3 & 31.983 & 0,2 & 1,2 \\
\hline $21-30$ & 7.877 & 0,1 & 13.564 & 0,1 & 0,6 \\
\hline $31-40$ & 20.211 & 0,2 & 19.431 & 0,1 & 1,0 \\
\hline $41-50$ & 26.859 & 0,2 & 17.730 & 0,1 & 1,5 \\
\hline $51-60$ & 214.282 & 1,7 & 140.584 & 0,8 & 1,5 \\
\hline 61-70 & 210.353 & 1,7 & 130.715 & 0,8 & 1,6 \\
\hline $71-80$ & 51.754 & 0,4 & 32.239 & 0,2 & 1,6 \\
\hline $81-90$ & 30.868 & 0,3 & 26.150 & 0,2 & 1,2 \\
\hline 91-100 & 63.775 & 0,5 & 63.660 & 0,4 & 1,0 \\
\hline 101-110 & 30.009 & 0,2 & 32.442 & 0,2 & 0,9 \\
\hline 111-120 & 602.787 & 4,9 & 817.673 & 4,9 & 0,7 \\
\hline 121-130 & 405.272 & 3,3 & 586.968 & 3,5 & 0,7 \\
\hline 131-140 & 961.662 & 7,8 & 1.407 .355 & 8,5 & 0,7 \\
\hline 141-150 & 524.775 & 4,3 & 803.936 & 4,8 & 0,7 \\
\hline 151-160 & 633.429 & 5,2 & 1.315 .982 & 7,9 & 0,5 \\
\hline 161-170 & 242.333 & 2,0 & 324.812 & 1,9 & 0,7 \\
\hline 171-180 & 824.017 & 6,7 & 1.197 .084 & 7,2 & 0,7 \\
\hline 181-190 & 292.149 & 2,4 & 374.768 & 2,3 & 0,8 \\
\hline 191-200 & 1.517 .957 & 12,4 & 2.219 .672 & 13,3 & 0,7 \\
\hline 201- - & 5.582 .382 & 45,4 & 7.085 .854 & 42,6 & 0,8 \\
\hline Totais & 12.282 .413 & 100,0 & 16.642 .772 & 100,0 & 0,7 \\
\hline
\end{tabular}


TABELA 3

Distribuição relativa (\%) da produção de pescado, segundo as principais espécies capturadas por barcos linheiros em águas tropicais $\left(18^{\circ}-23^{\circ} \mathrm{S}\right)$ e subtropicais $\left(23^{\circ}-26^{\circ} \mathrm{S}\right)$ ao largo da costa sudeste do Brasil, em cada faixa de latitude, no período 1986-1995.

\begin{tabular}{|c|c|c|c|c|c|c|c|}
\hline \multirow{3}{*}{$\begin{array}{l}\text { Latitudes } \\
\quad\left({ }^{\circ} \mathbf{S}\right)\end{array}$} & \multicolumn{7}{|c|}{ Produção de pescado (\%) } \\
\hline & \multicolumn{5}{|c|}{ Principais espécies } & \multirow{2}{*}{$\begin{array}{l}\text { Outras } \\
\text { espécies }\end{array}$} & \multirow{2}{*}{ Total } \\
\hline & Badejo & Batata & Cherne & Garoupa & Namorado & & \\
\hline \multicolumn{8}{|c|}{ * Águas tropicais $\left(18^{\circ}-23^{\circ} \mathrm{S}\right)$} \\
\hline 18-19 & 23,6 & 0,2 & 0,8 & 33,3 & 0,0 & 42,1 & 100,0 \\
\hline $19-20$ & 29,5 & 0,3 & 0,4 & 36,1 & 0,1 & 33,6 & 100,0 \\
\hline 20-21 & 13,0 & 14,5 & 50,5 & 1,0 & 2,6 & 18,4 & 100,0 \\
\hline 21-22 & 1,7 & 9,7 & 5,3 & 0,4 & 2,8 & 80,1 & 100,0 \\
\hline $22-23$ & 0,2 & 21,5 & 22,7 & 1,1 & 15,6 & 38,9 & 100,0 \\
\hline Totais & 12,3 & 6,1 & 5,4 & 15,7 & 2,3 & 58,2 & 100,0 \\
\hline \multicolumn{8}{|c|}{ * Águas subtropicais $\left(23^{\circ}-26^{\circ} \mathrm{S}\right)$} \\
\hline 23-24 & 0,4 & 28,7 & 15,3 & 0,7 & 25,1 & 29,8 & 100,0 \\
\hline $24-25$ & 0,3 & 56,4 & 12,5 & 0,5 & 20,9 & 9,4 & 100,0 \\
\hline $25-26$ & 0,3 & 59,7 & 12,0 & 0,8 & 21,9 & 5,3 & 100,0 \\
\hline Totais & 0,3 & 42,4 & 14,3 & 0,7 & 23,7 & 18,6 & 100,0 \\
\hline
\end{tabular}

- existem dois agrupamentos de espécies dominantes, um em águas tropicais e o outro em águas subtropicais, que podem ser tomados como duas guildas (Austen et al., 1994);

- no conjunto das capturas efetuadas nas águas tropicais, o agrupamento dominante é formado pela garoupa e o badejo, o que se mostrou de forma indiscutível entre as latitudes $18^{\circ}-20^{\circ} \mathrm{S}$;

- entre as latitudes $20^{\circ}-23^{\circ} \mathrm{S}$, portanto, ainda em águas tropicais, a dominância observada correspondeu às outras espécies estudadas, bem evidente na faixa compreendida entre as latitudes $22^{\circ}-23^{\circ} \mathrm{S}$, onde a dominância ficou com o cherne, o batata e o namorado, na ordem decrescente das capturas;

- os elevados valores das capturas do conjunto formado por "outras" espécies, nas águas tropicais, é seguro indicativo da grande diversidade de peixes na biocenose bentônica tropical;

- nas águas tropicais, as maiores capturas da garoupa e do badejo ocorreram em profundidades de até 80 metros, caracterizando a dominância das duas espécies no conjunto dos peixes bentônicos da parte interna da plataforma continental;

- o agrupamento dominante nas águas subtropicais foi sempre formado pelo batata, o namorado e o cherne, com maiores capturas em profundidades superiores a 100 metros, caracterizando a ictiofauna bentônica das partes externas da plataforma e superior do talude continentais;

- a progressiva redução das capturas do conjunto formado por "outras" espécies, nas águas subtropicais, e com o aumento da latitude, comprovou-se a menor diversidade de peixes na biocenose bentônica subtropical, mais acentuada no sul da área considerada.

A garoupa e o badejo, juntamente com a cioba (yellowtail snapper) = Ocyurus chrysurus $($ Bloch $)$, são as espécies dominantes na biomassa de peixes bentônicos da área de Abrolhos (Brasil), situada imediatamente ao norte da área estudada neste trabalho (Paiva \& Fonteles-Filho, 1995).

Badejo - Ocorre ao largo de toda a costa sudeste do Brasil, concentrando-se nas suas águas tropicais, principalmente entre as latitudes de $18^{\circ}$ $20^{\circ} \mathrm{S}$, onde se efetivaram $83,1 \%$ das capturas e 
foram registrados os melhores índices de abundância $(0,5 \mathrm{~kg} / \mathrm{anzol} / \mathrm{dia})$; as maiores capturas ocorreram no primeiro semestre (verão-outono), decrescendo a seguir; a produção e a produtividade das pescarias tenderam a decrescer com o aumento das latitudes; não foram evidentes deslocamentos estacionais ao longo da costa (Tabelas 3 e 6-7). Suas maiores capturas e melhores índices de abundância, tanto em águas tropicais como subtropicais, corresponderam a pescarias efetuadas em profundidades de até 80 metros, embora tenha sido encontrado em toda largura/profundidade da plataforma e mesmo no talude continentais, neste último com capturas registradas a até a 360 metros de profundidade; os índices de abundância tenderam a decrescer com o aumento da profundidade e foram muito baixos a partir de 80 metros nas águas tropicais e de 20 metros nas águas subtropicais (Tabelas 4-5 e 16-17).

Batata - Ocorre ao largo de toda a costa sudeste do Brasil, concentrando-se nas suas águas subtropicais, principalmente entre as latitudes de $23^{\circ}-25^{\circ} \mathrm{S}$, onde se efetivaram $92,7 \%$ das capturas, com índices maiores de abundância entre as latitudes $24^{\circ}-26^{\circ}(0,4-0,5 \mathrm{~kg} / \mathrm{anzol} / \mathrm{dia})$; as melhores capturas foram realizadas no primeiro semestre (verão-outono), decrescendo a seguir; a produção e a produtividade tenderam a crescer com o aumento das latitudes, até o limite de $25^{\circ} \mathrm{S}$; não foram evidentes deslocamentos estacionais ao longo da costa (Tabelas 3 e 8-9). Suas maiores capturas e melhores índices de abundância, tanto em águas tropicais como subtropicais, corresponderam a pesqueiros mais profundos, normalmente com mais de 100 metros de profundidade, e mesmo àqueles situados no talude continental (águas subtropicais), embora tenha sido encontrado em profundidades pouco superiores a 10 metros, ao longo da costa regional (Tabelas 4-5 e 16-17). No talude continental, capturas foram registradas em profundidades de até 1.500 metros.

Cherne - Ocorre ao largo de toda a costa sudeste do Brasil, concentrando-se nas suas águas subtropicais, entre as latitudes $23^{\circ}-25^{\circ} \mathrm{S}$, onde se efetivaram $86,1 \%$ das capturas, porém o índice máximo de abundância foi registrado entre as latitudes $20^{\circ}-21^{\circ} \mathrm{S}(0,5 \mathrm{~kg} / \mathrm{anzol} / \mathrm{dia})$; houve decréscimo das capturas no decorrer do ano, que foram maiores no primeiro trimestre (verão); a produção tendeu a crescer com o aumento das latitudes, até o limite de $25^{\circ} \mathrm{S}$, o mesmo não acon- tecendo com a produtividade, que ficou praticamente estável entre as latitudes $21^{\circ}-26^{\circ} \mathrm{S}$; os dados sugerem deslocamento estacional, no terceiro trimestre (inverno), da faixa $23^{\circ}-24^{\circ} \mathrm{S}$ para a seguinte, mais ao sul, com retorno no quarto trimestre (primavera) (Tabelas 3 e 10-11). Nas águas tropicais, suas maiores capturas ocorreram entre as profundidades de 111-130 metros e de 191-200 metros, porém os melhores índices de abundância ficaram restritos às profundidades de 71-100 metros, tendo sido pescado apenas em profundidades superiores a 30 metros; em águas subtropicais, ocorreu em toda a largura/profundidade da plataforma e parte superior do talude continentais, com maiores capturas entre 131-140 e 191200 metros de profundidade, concentrando-se no talude continental, porém os melhores índices de abundância ficaram restritos às profundidades de 61-110 metros (desconsiderando o registrado em águas com até 10 metros de profundidade); em ambas as massas d'água, os índices de abundância tenderam à estabilização, antes e depois das faixas de profundidade em que foram obtidos aqueles mais elevados (Tabelas 4-5 e 16-17). No talude continental, as capturas ocorreram em profundidades de até 630 metros.

Garoupa - Ocorre ao largo de toda a costa sudeste do Brasil, concentrando-se nas suas águas tropicais, entre as latitudes $18^{\circ}-20^{\circ} \mathrm{S}$, onde se efetivaram $86,4 \%$ das capturas, com os melhores índices de abundância (0,6-0,7 kg/anzol/dia); as maiores capturas foram realizadas no segundo e terceiro trimestres (outono-inverno), principalmente no segundo, com as mais baixas no quarto trimestre (primavera); após as faixas de concentração da produção, houve duas outras de importância secundária $\left(23^{\circ}-25^{\circ} \mathrm{S}\right)$, o mesmo não acontecendo com a produtividade das pescarias, que se manteve muito baixa; os dados não sugerem deslocamentos estacionais ao longo da costa (Tabelas 3 e 12 13). Nas águas tropicais, as maiores capturas ocorreram entre 31-60 metros de profundidade, com bons índices de abundância em pescarias realizadas entre 11-80 metros de profundidade, permanecendo muito baixos nos pesqueiros mais profundos; nas águas subtropicais, as melhores pescarias ficaram restritas a profundidades de 21-60 e 171-200 metros e no talude continental, com índices de abundância sempre muito baixos, alcançando somente $0,1 \mathrm{~kg} / \mathrm{anzol} / \mathrm{dia}$ entre $21-40$ metros de profundidade (Tabelas 4-5 e 16-17). No talu- 
de continental, foram registradas capturas em profundidades de até 300 metros.

Namorado - Ocorre ao longo de toda a costa sudeste do Brasil, concentrando-se nas suas águas subtropicais, entre as latitudes $23^{\circ}-25^{\circ} \mathrm{S}$, onde se efetivaram $92,9 \%$ das capturas, com os mais altos índices de abundância $(0,2 \mathrm{~kg} / \mathrm{anzol} /$ dia) correspondendo às latitudes $22^{\circ}-25^{\circ} \mathrm{S}$; as maiores capturas foram realizadas no primeiro trimestre (verão) e as menores, no segundo trimestre (outono), tendo havido um certo equilíbrio nos dois últimos trimestres (inverno-primavera); as faixas de latitude imediatamente abaixo e acima daquelas de maior produtividade apresentaram índices de abundância de $0,1 \mathrm{~kg} / \mathrm{anzol} / \mathrm{dia}$, porém, nas demais, estes índices foram extremamente bai- xos; os dados não sugerem deslocamentos estacionais ao longo da costa (Tabelas 3 e 14-15). Nas águas tropicais, suas melhores capturas foram efetivadas entre as profundidades de 121-140 e 171180 metros, com as maiores entre 191-200 metros, sendo normalmente baixos os índices de abundância em toda a amplitude de profundidade dos pesqueiros, com o máximo de $0,2 \mathrm{~kg} / \mathrm{anzol} /$ dia entre 81-90 metros de profundidade; nas águas subtropicais, suas melhores capturas começaram a ocorrer em pesqueiros com mais de 110 metros de profundidade, concentrando-se a partir de 191 metros, com índices de abundância quase sempre iguais a 0,2 kg/anzol/dia (Tabelas $4-5$ e 16-17). Foram realizadas capturas em profundidades de até 1.500 metros, no talude continental.

TABELA 4

Distribuição relativa (\%) da produção total de pescado, segundo as principais espécies capturadas por barcos linheiros em águas tropicais $\left(1^{\circ}-23^{\circ} \mathrm{S}\right)$ ao largo da costa sudeste do Brasil, em cada faixa de profundidade, no período 1986-1995.

\begin{tabular}{|c|c|c|c|c|c|c|c|}
\hline \multirow{3}{*}{$\begin{array}{l}\text { Profundidades } \\
\text { (m) }\end{array}$} & \multicolumn{7}{|c|}{ Produção de pescado $(\%)$} \\
\hline & \multicolumn{5}{|c|}{ Principais espécies } & \multirow{2}{*}{$\begin{array}{c}\text { Outras } \\
\text { espécies }\end{array}$} & \multirow{2}{*}{ Total } \\
\hline & Badejo & Batata & Cherne & Garoupa & Namorado & & \\
\hline--10 & 33,2 & - & - & - & - & 66,8 & 100,0 \\
\hline 11-20 & 26,7 & 0,2 & - & 7,4 & 0,4 & 65,3 & 100,0 \\
\hline 21-30 & 22,2 & 0,4 & - & 24,0 & - & 53,4 & 100,0 \\
\hline $31-40$ & 21,7 & 0,3 & 0,2 & 33,0 & - & 44,8 & 100,0 \\
\hline $41-50$ & 21,7 & 0,0 & 1,0 & 38,5 & 0,0 & 38,8 & 100,0 \\
\hline $51-60$ & 26,6 & 0,2 & 0,6 & 34,5 & 0,0 & 38,1 & 100,0 \\
\hline 61-70 & 26,7 & - & 0,9 & 35,2 & 0,3 & 36,9 & 100,0 \\
\hline 71-80 & 28,9 & 0,2 & 15,1 & 21,4 & 3,4 & 31,0 & 100,0 \\
\hline $81-90$ & - & 17,4 & 30,3 & - & 8,3 & 44,0 & 100,0 \\
\hline 91-100 & 2,1 & 10,2 & 16,7 & 9,8 & 2,7 & 58,5 & 100,0 \\
\hline $101-110$ & 2,1 & 5,8 & 7,6 & 0,4 & 2,3 & 81,8 & 100,0 \\
\hline $111-120$ & 4,1 & 5,3 & 4,4 & 0,4 & 1,8 & 84,0 & 100,0 \\
\hline $121-130$ & 1,4 & 5,5 & 4,5 & 0,2 & 2,7 & 85,7 & 100,0 \\
\hline $131-140$ & 0,2 & 20,6 & 10,9 & - & 9,7 & 58,6 & 100,0 \\
\hline 141-150 & 2,6 & 16,8 & 26,4 & 1,6 & 18,9 & 33,7 & 100,0 \\
\hline 151-160 & 2,4 & 3,6 & 4,2 & 0,5 & 1,8 & 87,5 & 100,0 \\
\hline $161-170$ & - & 32,0 & 20,7 & - & 10,4 & 36,9 & 100,0 \\
\hline 171-180 & 2,4 & 5,1 & 6,9 & 0,2 & 6,8 & 78,6 & 100,0 \\
\hline 181-190 & 1,3 & 0,6 & 2,9 & - & 1,9 & 93,3 & 100,0 \\
\hline 191-200 & 4,0 & 22,0 & 21,6 & 3,1 & 11,0 & 38,3 & 100,0 \\
\hline 201-- & - & - & - & - & - & - & - \\
\hline Totais & 16,3 & 3,2 & 3,5 & 20,6 & 1,7 & 54,7 & 100,0 \\
\hline
\end{tabular}


TABELA 5

Distribuição relativa (\%) da produção total de pescado, segundo as principais espécies capturadas por barcos linheiros em águas subtropicais $\left(23^{\circ}-26^{\circ} \mathrm{S}\right)$ ao largo da costa sudeste do Brasil, por faixas de profundidade, no período 1986-1995.

\begin{tabular}{|c|c|c|c|c|c|c|c|}
\hline \multirow{3}{*}{$\begin{array}{c}\text { Profundidades } \\
\text { (m) }\end{array}$} & \multicolumn{7}{|c|}{ Produção de pescado $(\%)$} \\
\hline & \multicolumn{5}{|c|}{ Principais espécies } & \multirow{2}{*}{$\begin{array}{l}\text { Outras } \\
\text { espécies }\end{array}$} & \multirow{2}{*}{ Total } \\
\hline & Badejo & Batata & Cherne & Garoupa & Namorado & & \\
\hline--10 & - & - & 4,8 & - & 0,3 & 94,9 & 100,0 \\
\hline 11-20 & 23,3 & 22,6 & 4,7 & 1,2 & 15,0 & 33,2 & 100,0 \\
\hline 21-30 & - & 19,7 & 6,3 & 10,2 & 18,2 & 45,6 & 100,0 \\
\hline $31-40$ & 5,1 & 0,8 & 6,1 & 6,5 & 17,6 & 63,9 & 100,0 \\
\hline $41-50$ & - & 5,0 & 7,0 & 1,4 & 12,1 & 74,5 & 100,0 \\
\hline $51-60$ & 0,5 & 4,1 & 6,7 & 0,6 & 4,6 & 83,5 & 100,0 \\
\hline $61-70$ & 0,0 & 6,2 & 9,4 & 0,2 & 8,5 & 75,7 & 100,0 \\
\hline 71-80 & 0,2 & 4,4 & 19,7 & 0,2 & 11,7 & 63,8 & 100,0 \\
\hline $81-90$ & - & 5,2 & 19,5 & - & 18,9 & 56,4 & 100,0 \\
\hline $91-100$ & 0,0 & 7,1 & 15,1 & - & 26,7 & 51,1 & 100,0 \\
\hline 101-110 & - & 11,3 & 18,6 & - & 27,4 & 42,7 & 100,0 \\
\hline 111-120 & 0,2 & 15,1 & 12,8 & 0,1 & 25,6 & 46,2 & 100,0 \\
\hline 121-130 & 0,0 & 20,7 & 14,0 & 0,1 & 29,8 & 35,4 & 100,0 \\
\hline 131-140 & 0,0 & 22,0 & 17,3 & - & 31,0 & 29,7 & 100,0 \\
\hline 141-150 & 0,1 & 23,4 & 16,2 & - & 34,7 & 25,6 & 100,0 \\
\hline $151-160$ & 0,0 & 27,9 & 18,2 & 0,0 & 36,0 & 17,9 & 100,0 \\
\hline $161-170$ & - & 22,4 & 18,8 & - & 31,5 & 27,3 & 100,0 \\
\hline 171-180 & 0,0 & 32,6 & 16,8 & 0,2 & 31,7 & 18,7 & 100,0 \\
\hline 181-190 & - & 35,7 & 13,4 & 0,3 & 28,9 & 21,7 & 100,0 \\
\hline 191-200 & 0,8 & 46,0 & 14,7 & 0,1 & 25,6 & 12,8 & 100,0 \\
\hline 201- - & 0,0 & 60,0 & 9,6 & 0,1 & 21,0 & 9,3 & 100,0 \\
\hline Totais & 0,2 & 42,4 & 12,6 & 0,1 & 24,8 & 19,9 & 100,0 \\
\hline
\end{tabular}

\section{CONCLUSÕES}

1. As principais espécies explotadas pela frota de linheiros, ao largo da costa sudeste do Brasil (1986-1995), contribuíram com 71,1\% das capturas, na seguinte ordem de importância decrescente: batata $=33,4 \%$, namorado $=18,4 \%$, cherne $=12,1 \%$, garoupa $=4,1 \%$ e badejo $=$ $3,1 \%$.

2. As pescarias da frota de linheiros, ao largo da costa sudeste do Brasil, são grandemente concentradas em suas águas subtropicais, com tendência(s) de produtividade decrescente, à medida que aumenta(m) a latitude e/ou a profundidade dos pesqueiros explotados.
3. Em geral, as espécies estudadas preferem águas tropicais ou subtropicais, o que é bem evidente na distribuição de suas capturas pela frota de linheiros em operação ao largo da costa sudeste do Brasil.

4. As cinco espécies estudadas constituem dois grupos dominantes na biomassa dos peixes bentônicos do sudeste do Brasil, um em águas tropicais e o outro em águas subtropicais.

5. Nas águas tropicais, o agrupamento dominante é formado pela garoupa e o badejo, o que se mostrou de forma indiscutível entre as latitudes $18^{\circ}-20^{\circ} \mathrm{S}$. Mais ao sul, houve dominância das outras espécies estudadas, num processo de transição para as águas subtropicais. 
TABELA 6

Distribuição relativa (\%) da produção e da produtividade (CPUE = kg/anzoldia) do badejo, nas pescarias de barcos linheiros ao largo da costa sudeste do Brasil, por trimestres e faixas de latitude, no período 1986-1995.

\begin{tabular}{|c|c|c|c|c|c|c|c|c|c|c|c|}
\hline \multirow{3}{*}{$\begin{array}{l}\text { Latitudes } \\
\quad\left({ }^{\circ} \mathbf{S}\right)\end{array}$} & \multicolumn{8}{|c|}{ Trimestres } & \multirow{2}{*}{\multicolumn{3}{|c|}{ Totais das capturas }} \\
\hline & \multicolumn{2}{|c|}{$1^{\circ}$} & \multicolumn{2}{|c|}{29} & \multicolumn{2}{|c|}{$3^{\circ}$} & \multicolumn{2}{|c|}{$4^{\circ}$} & & & \\
\hline & $\%$ & CPUE & $\%$ & CPUE & $\%$ & CPUE & $\%$ & CPUE & kg & $\%$ & CPUE \\
\hline $18-19$ & 32,7 & 0,4 & 28,7 & 0,5 & 25,6 & 0,8 & 13,0 & 0,9 & 345.662 & 100,0 & 0,5 \\
\hline $19-20$ & 25,6 & 0,6 & 34,7 & 0,6 & 28,1 & 0,3 & 11,6 & 0,9 & 72.872 & 100,0 & 0,5 \\
\hline $20-21$ & - & - & 10,1 & 0,0 & - & - & 89,9 & 2,4 & 13.350 & 100,0 & 0,1 \\
\hline 21-22 & 30,0 & 0,1 & 13,1 & 0,0 & 29,4 & 0,1 & 27,5 & 0,1 & 28.225 & 100,0 & 0,0 \\
\hline $22-23$ & - & - & - & - & 100,0 & 0,0 & - & - & 490 & 100,0 & 0,0 \\
\hline 23-24 & 50,3 & 0,0 & 47,7 & 0,0 & 1,2 & 0,0 & 0,8 & 0,0 & 28.675 & 100,0 & 0,0 \\
\hline $24-25$ & - & - & 47,4 & 0,0 & 52,6 & 0,0 & - & - & 12.925 & 100,0 & 0,0 \\
\hline $25-26$ & - & - & 100,0 & 0,0 & - & - & - & - & 1.560 & 100,0 & 0,0 \\
\hline Totais & 30,7 & 0,0 & 29,9 & 0,0 & 24,8 & 0,0 & 14,6 & 0,0 & 503.759 & 100,0 & 0,0 \\
\hline
\end{tabular}

TABELA 7

Distribuição relativa (\%) trimestral e anual da produção do badejo, nas pescarias de barcos linheiros ao largo da costa sudeste do Brasil, entre as faixas de latitude, no período 1986-1995.

\begin{tabular}{|c|c|c|c|c|c|}
\hline \multirow{3}{*}{$\begin{array}{l}\text { Latitudes } \\
\quad\left({ }^{\circ} \mathbf{S}\right)\end{array}$} & \multicolumn{5}{|c|}{ Produção do badejo (\%) } \\
\hline & \multicolumn{4}{|c|}{ Trimestres } & \multirow{2}{*}{ Ano } \\
\hline & $1^{0}$ & $2^{-9}$ & $3^{\mathbf{0}}$ & $4^{0}$ & \\
\hline 18-19 & 73,2 & 65,7 & 70,8 & 61,2 & 68,5 \\
\hline $19-20$ & 12,0 & 16,8 & 16,4 & 11,5 & 14,5 \\
\hline $20-21$ & - & 0,9 & - & 16,4 & 2,6 \\
\hline 21-22 & 5,5 & 2,4 & 6,6 & 10,6 & 5,6 \\
\hline $22-23$ & - & - & 0,4 & - & 0,1 \\
\hline 23-24 & 9,3 & 9,1 & 0,3 & 0,3 & 5,7 \\
\hline 24-25 & - & 4,1 & 5,5 & - & 2,6 \\
\hline $25-26$ & - & 1,0 & - & - & 0,3 \\
\hline Totais & 100,0 & 100,0 & 100,0 & 100,0 & 100,0 \\
\hline
\end{tabular}

TABELA 8

Distribuição relativa (\%) da produção e da produtividade (CPUE = kg/anzol/dia) do batata, nas pescarias de barcos linheiros ao largo da costa sudeste do Brasil, por trimestres e faixas de latitude, no período 1986-1995.

\begin{tabular}{|c|c|c|c|c|c|c|c|c|c|c|c|}
\hline \multirow{3}{*}{$\begin{array}{l}\text { Latitudes } \\
\quad\left({ }^{\circ} \mathbf{S}\right)\end{array}$} & \multicolumn{8}{|c|}{ Trimestres } & \multirow{2}{*}{\multicolumn{3}{|c|}{ Totais das capturas }} \\
\hline & \multicolumn{2}{|c|}{$1^{0}$} & \multicolumn{2}{|c|}{$2^{\mathbf{o}}$} & \multicolumn{2}{|c|}{$3^{0}$} & \multicolumn{2}{|c|}{$4^{0}$} & & & \\
\hline & $\%$ & CPUE & $\%$ & CPUE & $\%$ & CPUE & $\%$ & CPUE & kg & $\%$ & CPUE \\
\hline $18-19$ & 34,4 & 0,0 & 7,3 & 0,0 & - & - & 58,3 & 0,0 & 3.020 & 100,0 & 0,0 \\
\hline $19-20$ & 79,4 & 0,0 & 20,6 & 0,0 & - & - & - & - & 630 & 100,0 & 0,0 \\
\hline $20-21$ & 57,7 & 0,4 & 34,0 & 0,1 & 5,0 & 0,0 & 3,3 & 0,1 & 14.910 & 100,0 & 0,2 \\
\hline $21-22$ & 21,3 & 0,2 & 68,4 & 0,4 & 8,5 & 0,1 & 5,8 & 0,0 & 165.955 & 100,0 & 0,2 \\
\hline $22-23$ & 51,9 & 0,3 & 16,6 & 0,2 & 9,3 & 0,1 & 22,2 & 0,2 & 43.765 & 100,0 & 0,2 \\
\hline 23-24 & 30,6 & 0,2 & 34,5 & 0,3 & 18,5 & 0,2 & 16,4 & 0,1 & 1.969 .398 & 100,0 & 0,2 \\
\hline 24-25 & 30,3 & 0,6 & 27,9 & 0,6 & 27,9 & 0,4 & 13,9 & 0,3 & 2.916 .125 & 100,0 & 0,5 \\
\hline $25-26$ & 36,7 & 0,6 & 28,7 & 0,4 & 22,8 & 0,5 & 11,8 & 0,1 & 356.777 & 100,0 & 0,4 \\
\hline Totais & 30,8 & 0,3 & 31,5 & 0,3 & 23,4 & 0,3 & 14,3 & 0,2 & 5.470 .580 & 100,0 & 0,3 \\
\hline
\end{tabular}


TABELA 9

Distribuição relativa (\%) trimestral e anual da produção do batata, nas pescarias de barcos linheiros ao largo da costa sudeste do Brasil, entre as faixas de latitude, no período 1986-1995.

\begin{tabular}{|c|c|c|c|c|c|}
\hline \multirow{3}{*}{$\begin{array}{l}\text { Latitudes } \\
\quad\left({ }^{\circ} \mathbf{S}\right)\end{array}$} & \multicolumn{5}{|c|}{ Produção do batata (\%) } \\
\hline & \multicolumn{4}{|c|}{ Trimestres } & \multirow{2}{*}{ Ano } \\
\hline & $1^{0}$ & $2^{0}$ & $3^{0}$ & $4^{0}$ & \\
\hline 18-19 & 0,1 & 0,0 & - & 0,2 & 0,1 \\
\hline $19-20$ & 0,0 & 0,0 & - & - & 0,0 \\
\hline $20-21$ & 0,5 & 0,3 & 0,1 & 0,1 & 0,3 \\
\hline 21-22 & 2,1 & 6,6 & 1,1 & 0,4 & 3,0 \\
\hline $22-23$ & 1,3 & 0,4 & 0,3 & 1,2 & 0,8 \\
\hline 23-24 & 35,8 & 39,4 & 28,5 & 41,2 & 36,0 \\
\hline 24-25 & 52,4 & 47,3 & 63,6 & 51,5 & 53,3 \\
\hline $25-26$ & 7,8 & 6,0 & 6,4 & 5,4 & 6,5 \\
\hline Totais & 100,0 & 100,0 & 100,0 & 100,0 & 100,0 \\
\hline
\end{tabular}

TABELA 10

Distribuição relativa (\%) da produção e da produtividade (CPUE $=\mathrm{kg} / \mathrm{anzol} / \mathrm{dia})$ do cherne, nas pescarias de barcos linheiros ao largo da costa sudeste do Brasil, por trimestres e faixas de latitude, no período 1986-1995.

\begin{tabular}{|c|c|c|c|c|c|c|c|c|c|c|c|}
\hline \multirow{3}{*}{$\begin{array}{c}\text { Latitudes } \\
\left({ }^{\circ} \mathbf{S}\right)\end{array}$} & \multicolumn{8}{|c|}{ Trimestres } & \multirow{2}{*}{\multicolumn{3}{|c|}{ Totais das capturas }} \\
\hline & \multicolumn{2}{|c|}{$1^{0}$} & \multicolumn{2}{|c|}{$2^{9}$} & \multicolumn{2}{|c|}{$3^{\circ}$} & \multicolumn{2}{|c|}{$4^{0}$} & & & \\
\hline & $\%$ & CPUE & $\%$ & CPUE & $\%$ & CPUE & $\%$ & CPUE & kg & $\%$ & CPUE \\
\hline 18-19 & 47,9 & 0,0 & 30,9 & 0,0 & 16,0 & 0,0 & 5,2 & 0,0 & 12.195 & 100,0 & 0,0 \\
\hline $19-20$ & - & - & - & - & 100,0 & 0,0 & - & - & 1.050 & 100,0 & 0,0 \\
\hline $20-21$ & 36,6 & 0,9 & 8,7 & 0,1 & 54,6 & 1,5 & 0,1 & 0,0 & 51.950 & 100,0 & 0,5 \\
\hline 21-22 & 40,9 & 0,2 & 41,4 & 0,1 & 13,0 & 0,1 & 4,7 & 0,0 & 90.903 & 100,0 & 0,1 \\
\hline $22-23$ & 60,6 & 0,4 & 16,9 & 0,2 & 9,7 & 0,1 & 12,8 & 0,1 & 46.378 & 100,0 & 0,2 \\
\hline 23-24 & 36,2 & 0,1 & 29,1 & 0,1 & 13,8 & 0,1 & 20,9 & 0,1 & 1.054 .102 & 100,0 & 0,1 \\
\hline $24-25$ & 24,9 & 0,1 & 23,9 & 0,1 & 30,1 & 0,1 & 21,1 & 0,1 & 646.826 & 100,0 & 0,1 \\
\hline $25-26$ & 16,0 & 0,1 & 42,0 & 0,1 & 16,8 & 0,1 & 25,2 & 0,1 & 71.705 & 100,0 & 0,1 \\
\hline Totais & 32,6 & 0,1 & 27,7 & 0,1 & 20,2 & 0,1 & 19,5 & 0,1 & 1.975 .109 & 100,0 & 0,1 \\
\hline
\end{tabular}

TABELA 11

Distribuição relativa (\%) trimestral e anual da produção do cherne, nas pescarias de barcos linheiros ao largo da costa sudeste do Brasil, entre as faixas de latitude, no período 1986-1995.

\begin{tabular}{|c|c|c|c|c|c|}
\hline \multirow{3}{*}{$\begin{array}{l}\text { Latitudes } \\
\quad\left({ }^{\circ} \mathrm{S}\right)\end{array}$} & \multicolumn{5}{|c|}{ Produção do cherne (\%) } \\
\hline & \multicolumn{4}{|c|}{ Trimestres } & \multirow{2}{*}{ Ano } \\
\hline & $1^{0}$ & $2^{-9}$ & $3^{-}$ & $4^{0}$ & \\
\hline 18-19 & 0,9 & 0,7 & 0,5 & 0,2 & 0,6 \\
\hline $19-20$ & - & - & 0,3 & - & 0,1 \\
\hline $20-21$ & 2,9 & 0,8 & 7,1 & 0,0 & 2,6 \\
\hline 21-22 & 5,8 & 6,9 & 3,0 & 1,1 & 4,6 \\
\hline $22-23$ & 4,3 & 1,5 & 1,1 & 1,5 & 2,4 \\
\hline $23-24$ & 59,3 & 56,2 & 36,3 & 57,1 & 53,4 \\
\hline 24-25 & 25,0 & 28,4 & 48,7 & 35,4 & 32,7 \\
\hline $25-26$ & 1,8 & 5,5 & 3,0 & 4,7 & 3,6 \\
\hline Totais & 100,0 & 100,0 & 100,0 & 100,0 & 100,0 \\
\hline
\end{tabular}


TABELA 12

Distribuição relativa (\%) da produção e da produtividade (CPUE = kg/anzol/dia) da garoupa, nas pescarias de barcos linheiros ao largo da costa sudeste do Brasil, por trimestres e faixas de latitude, no período 1986-1995.

\begin{tabular}{|c|c|c|c|c|c|c|c|c|c|c|c|}
\hline \multirow{3}{*}{$\begin{array}{l}\text { Latitudes } \\
\quad\left({ }^{\circ} \mathbf{S}\right)\end{array}$} & \multicolumn{8}{|c|}{ Trimestres } & \multirow{2}{*}{\multicolumn{3}{|c|}{ Totais das capturas }} \\
\hline & \multicolumn{2}{|c|}{$1^{0}$} & \multicolumn{2}{|c|}{$2^{-}$} & \multicolumn{2}{|c|}{$3^{-}$} & \multicolumn{2}{|c|}{$4^{\circ}$} & & & \\
\hline & $\%$ & CPUE & $\%$ & CPUE & $\%$ & CPUE & $\%$ & CPUE & kg & $\%$ & CPUE \\
\hline $18-19$ & 23,3 & 0,4 & 32,8 & 0,7 & 33,5 & 1,5 & 10,4 & 1,0 & 488.328 & 100,0 & 0,7 \\
\hline $19-20$ & 25,3 & 0,8 & 33,8 & 0,7 & 34,8 & 0,5 & 6,1 & 0,6 & 89.100 & 100,0 & 0,6 \\
\hline $20-21$ & - & - & 100,0 & 0,0 & - & - & - & - & 1.000 & 100,0 & 0,0 \\
\hline $21-22$ & 16,2 & 0,0 & 11,9 & 0,0 & 8,3 & 0,0 & 63,6 & 0,0 & 6.111 & 100,0 & 0,0 \\
\hline $22-23$ & - & - & - & - & 100,0 & 0,1 & - & - & 2.170 & 100,0 & 0,0 \\
\hline 23-24 & 33,3 & 0,0 & 62,2 & 0,0 & 2,1 & 0,0 & 2,4 & 0,0 & 49.702 & 100,0 & 0,0 \\
\hline $24-25$ & 0,4 & 0,0 & 77,6 & 0,0 & 22,0 & 0,0 & - & - & 26.485 & 100,0 & 0,0 \\
\hline $25-26$ & 2,0 & 0,0 & 98,0 & 0,0 & - & - & - & - & 5.000 & 100,0 & 0,0 \\
\hline Totais & 23,0 & 0,0 & 37,2 & 0,0 & 30,6 & 0,0 & 9,2 & 0,0 & 667.896 & 100,0 & 0,0 \\
\hline
\end{tabular}

TABELA 13

Distribuição relativa (\%) trimestral e anual da produção da garoupa, nas pescarias de barcos linheiros ao largo da costa sudeste do Brasil, entre as faixas de latitude, no período 1986-1995.

\begin{tabular}{|c|c|c|c|c|c|}
\hline \multirow{3}{*}{$\begin{array}{l}\text { Latitudes } \\
\quad\left({ }^{\circ} \mathbf{S}\right)\end{array}$} & \multicolumn{5}{|c|}{ Produção da garoupa $(\%)$} \\
\hline & \multicolumn{4}{|c|}{ Trimestres } & \multirow{2}{*}{ Ano } \\
\hline & $1^{0}$ & $2^{-}$ & $3^{0}$ & $4^{0}$ & \\
\hline $18-19$ & 73,8 & 64,5 & 80,2 & 82,8 & 73,1 \\
\hline $19-20$ & 14,7 & 12,1 & 15,2 & 8,9 & 13,3 \\
\hline 20-21 & - & 0,4 & - & - & 0,2 \\
\hline 21-22 & 0,6 & 0,3 & 0,2 & 6,3 & 0,9 \\
\hline $22-23$ & - & - & 1,1 & - & 0,3 \\
\hline 23-24 & 10,7 & 12,4 & 0,5 & 2,0 & 7,4 \\
\hline $24-25$ & 0,1 & 8,3 & 2,8 & - & 4,0 \\
\hline $25-26$ & 0,1 & 2,0 & - & - & 0,8 \\
\hline Totais & 100,0 & 100,0 & 100,0 & 100,0 & 100,0 \\
\hline
\end{tabular}

TABELA 14

Distribuição relativa (\%) da produção e da produtividade (CPUE = kg/anzol/dia) do namorado, nas pescarias de barcos linheiros ao largo da costa sudeste do Brasil, por trimestres e faixas de latitude, no período 1986-1995.

\begin{tabular}{|c|c|c|c|c|c|c|c|c|c|c|c|}
\hline \multirow{3}{*}{$\begin{array}{l}\text { Latitudes } \\
\quad\left({ }^{\circ} \mathbf{S}\right)\end{array}$} & \multicolumn{8}{|c|}{ Trimestres } & \multirow{2}{*}{\multicolumn{3}{|c|}{ Totais das capturas }} \\
\hline & \multicolumn{2}{|c|}{$1^{0}$} & \multicolumn{2}{|c|}{$2^{9}$} & \multicolumn{2}{|c|}{$3^{0}$} & \multicolumn{2}{|c|}{$4^{0}$} & & & \\
\hline & $\%$ & CPUE & $\%$ & CPUE & $\%$ & CPUE & $\%$ & CPUE & kg & $\%$ & CPUE \\
\hline 18-19 & 10,9 & 0,0 & 16,4 & 0,0 & 72,7 & 0,0 & - & - & 550 & 100,0 & 0,0 \\
\hline $19-20$ & - & - & - & - & 100,0 & 0,0 & - & - & 350 & 100,0 & 0,0 \\
\hline $20-21$ & 50,6 & 0,1 & 44,5 & 0,0 & 1,9 & 0,0 & 3,0 & 0,0 & 2.650 & 100,0 & 0,0 \\
\hline $21-22$ & 34,8 & 0,1 & 45,7 & 0,1 & 14,7 & 0,0 & 4,8 & 0,0 & 48.486 & 100,0 & 0,1 \\
\hline $22-23$ & 46,5 & 0,2 & 15,0 & 0,1 & 12,7 & 0,1 & 25,8 & 0,1 & 31.763 & 100,0 & 0,2 \\
\hline $23-24$ & 30,3 & 0,2 & 21,0 & 0,1 & 21,9 & 0,2 & 26,8 & 0,2 & 1.723 .976 & 100,0 & 0,2 \\
\hline $24-25$ & 25,4 & 0,2 & 22,1 & 0,2 & 29,5 & 0,1 & 23,0 & 0,2 & 1.083 .075 & 100,0 & 0,2 \\
\hline $25-26$ & 24,9 & 0,1 & 26,3 & 0,1 & 25,1 & 0,2 & 23,7 & 0,1 & 130.567 & 100,0 & 0,1 \\
\hline Totais & 28,6 & 0,2 & 21,9 & 0,1 & 24,6 & 0,2 & 24,9 & 0,2 & 3.021 .417 & 100,0 & 0,2 \\
\hline
\end{tabular}


TABELA 15

Distribuição relativa (\%) trimestral e anual da produção do namorado, nas pescarias de barcos linheiros ao largo da costa sudeste do Brasil, entre as faixas de latitude, no período 1986-1995.

\begin{tabular}{|c|c|c|c|c|c|}
\hline \multirow{3}{*}{$\begin{array}{l}\text { Latitudes } \\
\left({ }^{\circ} \mathbf{S}\right)\end{array}$} & \multicolumn{5}{|c|}{ Produção do namorado (\%) } \\
\hline & \multicolumn{4}{|c|}{ Trimestres } & \multirow{2}{*}{ Ano } \\
\hline & $1^{0}$ & $2^{-}$ & $3^{-}$ & $4^{0}$ & \\
\hline 18-19 & 0,0 & 0,0 & 0,1 & - & 0,0 \\
\hline $19-20$ & - & - & 0,1 & - & 0,0 \\
\hline $20-21$ & 0,2 & 0,2 & 0,0 & 0,0 & 0,1 \\
\hline 21-22 & 1,9 & 3,3 & 1,0 & 0,3 & 1,6 \\
\hline $22-23$ & 1,7 & 0,7 & 0,5 & 1,1 & 1,1 \\
\hline $23-24$ & 60,5 & 54,5 & 50,9 & 61,4 & 57,1 \\
\hline $24-25$ & 31,9 & 36,1 & 43,0 & 33,1 & 35,8 \\
\hline $25-26$ & 3,8 & 5,2 & 4,4 & 4,1 & 4,3 \\
\hline Totais & 100,0 & 100,0 & 100,0 & 100,0 & 100,0 \\
\hline
\end{tabular}

TABELA 16

Produção relativa (\%) e abundância $(C P U E=$ kg/anzol/dia) das principais espécies capturadas por barcos linheiros em águas tropicais $\left(1^{\circ}-23^{\circ} \mathrm{S}\right)$ ao largo da costa sudeste do Brasil, entre faixas de profundidade, no período 1986-1995.

\begin{tabular}{|c|c|c|c|c|c|c|c|c|c|c|}
\hline \multirow{3}{*}{$\begin{array}{l}\text { Profundidades } \\
\text { (m) }\end{array}$} & \multicolumn{10}{|c|}{ Produção e abundância das espécies } \\
\hline & \multicolumn{2}{|c|}{ Badejo } & \multicolumn{2}{|c|}{ Batata } & \multicolumn{2}{|c|}{ Cherne } & \multicolumn{2}{|c|}{ Garoupa } & \multicolumn{2}{|c|}{ Namorado } \\
\hline & $\%$ & CPUE & $\%$ & CPUE & $\%$ & CPUE & $\%$ & CPUE & $\%$ & CPUE \\
\hline--10 & 0,6 & 4,2 & - & - & - & - & - & - & - & - \\
\hline 11-20 & 6,0 & 1,8 & 0,2 & 0,0 & - & - & 1,3 & 0,5 & 0,8 & 0,0 \\
\hline 21-30 & 2,6 & 0,8 & 0,2 & 0,0 & - & - & 2,2 & 0,9 & - & - \\
\hline $31-40$ & 8,6 & 0,5 & 0,6 & 0,0 & 0,4 & 0,0 & 10,3 & 0,7 & - & - \\
\hline $41-50$ & 20,2 & 0,5 & 0,1 & 0,0 & 4,1 & 0,0 & 28,3 & 0,9 & 0,3 & 0,0 \\
\hline $51-60$ & 45,7 & 0,5 & 1,6 & 0,0 & 4,5 & 0,0 & 46,8 & 0,6 & 0,8 & 0,0 \\
\hline $61-70$ & 8,0 & 0,6 & - & - & 1,2 & 0,0 & 8,3 & 0,8 & 0,7 & 0,0 \\
\hline $71-80$ & 2,5 & 0,9 & 0,1 & 0,0 & 5,9 & 0,5 & 1,4 & 0,7 & 2,8 & 0,1 \\
\hline $81-90$ & - & - & 0,8 & 0,4 & 1,2 & 0,6 & - & - & 0,7 & 0,2 \\
\hline $91-100$ & 0,1 & 0,0 & 2,6 & 0,2 & 3,8 & 0,4 & 0,4 & 0,2 & 1,2 & 0,1 \\
\hline $101-110$ & 0,3 & 0,1 & 4,8 & 0,2 & 5,7 & 0,2 & 0,0 & 0,0 & 3,5 & 0,1 \\
\hline 111-120 & 2,1 & 0,1 & 13,8 & 0,2 & 10,5 & 0,1 & 0,1 & 0,0 & 8,9 & 0,1 \\
\hline 121-130 & 0,8 & 0,0 & 15,1 & 0,1 & 10,9 & 0,1 & 0,1 & 0,0 & 13,4 & 0,1 \\
\hline $131-140$ & 0,0 & 0,0 & 14,5 & 0,1 & 7,0 & 0,1 & - & - & 12,6 & 0,1 \\
\hline $141-150$ & 0,0 & 0,0 & 1,6 & 0,1 & 2,3 & 0,1 & 0,0 & 0,0 & 3,3 & 0,1 \\
\hline $151-160$ & 0,8 & 0,1 & 6,2 & 0,1 & 6,5 & 0,1 & 0,1 & 0,0 & 5,6 & 0,0 \\
\hline $161-170$ & - & - & 1,5 & 0,2 & 0,9 & 0,1 & - & - & 0,9 & 0,1 \\
\hline 171-180 & 0,6 & 0,0 & 6,5 & 0,1 & 7,9 & 0,1 & 0,0 & 0,0 & 16,0 & 0,1 \\
\hline $181-190$ & 0,1 & 0,0 & 0,2 & 0,0 & 1,0 & 0,1 & - & - & 1,4 & 0,0 \\
\hline $191-200$ & 1,0 & 0,0 & 29,6 & 0,2 & 26,2 & 0,2 & 0,7 & 0,0 & 27,1 & 0,1 \\
\hline 201-- & - & - & - & - & - & - & - & - & - & - \\
\hline Totais & 100,0 & 0,3 & 100,0 & 0,1 & 100,0 & 0,1 & 100,0 & 0,4 & 100,0 & 0,0 \\
\hline
\end{tabular}


TABELA 17

Produção relativa $(\%)$ e abundância $($ CPUE $=\mathrm{kg} / \mathrm{anzol} / \mathrm{dia})$ das principais espécies capturadas por barcos linheiros em águas subtropicais $\left(23^{\circ}-26^{\circ} \mathrm{S}\right)$ ao largo da costa sudeste do Brasil, entre faixas de profundidade, no período 1986-1995.

\begin{tabular}{|c|c|c|c|c|c|c|c|c|c|c|}
\hline \multirow{3}{*}{$\begin{array}{l}\text { Profundidades } \\
\text { (m) }\end{array}$} & \multicolumn{10}{|c|}{ Produção e abundância das espécies } \\
\hline & \multicolumn{2}{|c|}{ Badejo } & \multicolumn{2}{|c|}{ Batata } & \multicolumn{2}{|c|}{ Cherne } & \multicolumn{2}{|c|}{ Garoupa } & \multicolumn{2}{|c|}{ Namorado } \\
\hline & $\%$ & CPUE & $\%$ & CPUE & $\%$ & CPUE & $\%$ & CPUE & $\%$ & CPUE \\
\hline--10 & - & - & - & - & 0,0 & 0,5 & - & - & 0,0 & 0,0 \\
\hline $11-20$ & 34,2 & 0,3 & 0,2 & 0,3 & 0,1 & 0,1 & 3,0 & 0,0 & 0,2 & 0,2 \\
\hline 21-30 & - & - & 0,0 & 0,1 & 0,0 & 0,0 & 5,3 & 0,1 & 0,0 & 0,1 \\
\hline $31-40$ & 4,0 & 0,1 & 0,0 & 0,0 & 0,1 & 0,1 & 8,7 & 0,1 & 0,1 & 0,2 \\
\hline $41-50$ & - & - & 0,0 & 0,1 & 0,1 & 0,1 & 2,5 & 0,0 & 0,1 & 0,2 \\
\hline $51-60$ & 4,2 & 0,0 & 0,2 & 0,1 & 0,9 & 0,1 & 8,8 & 0,0 & 0,3 & 0,1 \\
\hline $61-70$ & 0,3 & 0,0 & 0,2 & 0,1 & 1,3 & 0,2 & 1,9 & 0,0 & 0,6 & 0,1 \\
\hline $71-80$ & 0,3 & 0,0 & 0,0 & 0,1 & 0,7 & 0,3 & 0,7 & 0,0 & 0,2 & 0,2 \\
\hline $81-90$ & - & - & 0,0 & 0,1 & 0,4 & 0,2 & - & - & 0,2 & 0,2 \\
\hline $91-100$ & 0,0 & 0,0 & 0,1 & 0,1 & 0,6 & 0,2 & - & - & 0,5 & 0,3 \\
\hline $101-110$ & - & - & 0,1 & 0,1 & 0,4 & 0,2 & - & - & 0,3 & 0,3 \\
\hline 111-120 & 3,9 & 0,0 & 1,8 & 0,1 & 5,0 & 0,1 & 3,0 & 0,0 & 5,0 & 0,2 \\
\hline 121-130 & 0,8 & 0,0 & 1,6 & 0,2 & 3,7 & 0,1 & 2,0 & 0,0 & 4,0 & 0,2 \\
\hline 131-140 & 0,2 & 0,0 & 4,1 & 0,2 & 10,7 & 0,1 & - & - & 9,8 & 0,2 \\
\hline 141-150 & 1,2 & 0,0 & 2,4 & 0,2 & 5,5 & 0,1 & - & - & 6,0 & 0,2 \\
\hline $151-160$ & 0,2 & 0,0 & 3,4 & 0,1 & 7,4 & 0,1 & 0,4 & 0,0 & 7,5 & 0,2 \\
\hline $161-170$ & - & - & 1,0 & 0,2 & 2,9 & 0,1 & - & - & 2,5 & 0,2 \\
\hline 171-180 & 0,8 & 0,0 & 5,2 & 0,2 & 8,9 & 0,1 & 9,5 & 0,0 & 8,6 & 0,2 \\
\hline 181-190 & - & - & 2,0 & 0,3 & 2,5 & 0,1 & 5,1 & 0,0 & 2,8 & 0,2 \\
\hline 191-200 & 46,0 & 0,0 & 13,4 & 0,3 & 14,4 & 0,1 & 10,7 & 0,0 & 12,8 & 0,2 \\
\hline 201-- & 3,9 & 0,0 & 64,3 & 0,5 & 34,4 & 0,1 & 38,4 & 0,0 & 38,5 & 0,2 \\
\hline Totais & 100,0 & 0,0 & 100,0 & 0,3 & 100,0 & 0,1 & 100,0 & 0,0 & 100,0 & 0,2 \\
\hline
\end{tabular}

6. Os elevados valores das capturas de "outras" espécies, nas águas tropicais, é seguro indicativo da grande diversidade de ictiofauna na biocenose bentônica tropical.

7. Nas águas tropicais, as maiores capturas da garoupa e do badejo ocorreram em profundidades de até 80 metros, caracterizando a dominância das duas espécies no conjunto dos peixes bentônicos da parte interna da plataforma continental.

8. O agrupamento dominante nas águas subtropicais foi sempre formado pelo batata, o namorado e o cherne, com maiores capturas em profundidades superiores a 100 metros, caracterizando a ictiofauna bentônica das partes externa da plataforma e superior do talude continentais.

9. A progressiva redução das capturas de "outras" espécies, nas águas subtropicais, com os aumentos da latitude e da profundidade, comprova a menor diversidade de peixes na biocenose bentônica subtropical.
10. O badejo prefere águas tropicais, concentrando-se entre as latitudes $18^{\circ}-20^{\circ} \mathrm{S}$ e em profundidades de até 80 metros, onde foram registrados os melhores índices de abundância, sem evidentes deslocamentos estacionais ao longo da costa.

11. O batata prefere águas subtropicais, concentrando-se entre as latitudes $23^{\circ}-25^{\circ} \mathrm{S}$ e em pesqueiros normalmente com mais de 100 metros de profundidade e mesmo no talude continental, onde foram registrados os melhores índices de abundância, sem evidentes deslocamentos estacionais ao longo da costa.

12. O cherne prefere águas subtropicais, concentrando-se entre as latitudes $23^{\circ}-25^{\circ} \mathrm{S}$, com índice máximo de abundância entre as latitudes $20^{\circ}-21^{\circ} \mathrm{S}$ (águas tropicais); nas águas subtropicais, suas melhores capturas se efetuaram em pesqueiros com mais de 130 metros de fundura, principalmente na parte superior do talude continental; os dados sugerem deslocamen- 
to durante o inverno, da faixa de $23^{\circ}-24^{\circ} \mathrm{S}$ para a seguinte mais ao sul, com retorno na primavera.

13. A garoupa prefere águas tropicais, concentrando-se entre as latitudes $18^{\circ}-20^{\circ} \mathrm{S}$ e em profundidades de até 80 metros, onde foram registrados os melhores índices de abundância, sem evidentes deslocamentos ao longo da costa.

14. O namorado prefere águas subtropicais, concentrando-se entre as latitudes $23^{\circ}-25^{\circ} \mathrm{S}$ e em pesqueiros com mais de 190 metros de fundura, na plataforma e talude continentais, com certa estabilização dos índices de abundância a partir de 80 metros de profundidade; os dados não sugerem deslocamentos estacionais ao longo da costa.

Agradecimentos: Somos gratos ao Instituto Brasileiro do Meio Ambiente e dos Recursos Naturais Renováveis (IBAMA), por haver permitido a utilização dos dados que suportam o presente trabalho. Também agradecemos os apoios recebidos da Universidade Federal do Rio de Janeiro (UFRJ) e do Conselho Nacional de Desenvolvimento Científico e Tecnológico (CNPq).

\section{REFERÊNCIAS BIBLIOGRÁFICAS}

ANDRADE, M. F., 1995, Distribuição e abundância de alguns peixes bentônicos na plataforma continental do sudeste do Brasil. Monografia de Graduação, UFRJ Departamento de Biologia Marinha, Rio de Janeiro, 37p.

AUSTEN, D. J., BAYLEY, P. B. \& MENZEL, B. W., 1994, Importance of the Guild Concept to Fisheries Research and Management. Fisheries, Bethesda, 19(6): 12-20.

BRIGGS, J. C., 1974, Marine Zoogeography. McGraw-Hill Book Company, New York, 489p.

CARVAlHO FILHO, A., 1992, Peixes: costa brasileira. Editora Marca d'Água, 304p.

FIGUEIREDO, J. L. \& MENEZES, N. A., 1980, Manual de peixes marinhos do sudeste do Brasil. III. Teleostei (2). Universidade de São Paulo/Museu de Zoologia, São Paulo, 94p

HAIMOVICI, M., MARTINS, A. S. \& VIEIRA, P. C., 1996, Distribuição e abundância de peixes teleósteos demersais sobre a plataforma continental do sul do Brasil. Rev. Brasil. Biol., Rio de Janeiro, 56(1): 27-50.

HEEMSTRA, P. C. \& RANDALL, J. E., 1993, Groupers of the world (Family Serranidae, Subfamily Epinephelinae). An annotaded and illustrated catalogue of the grouper, rockcod, hind, coral grouper and lyretail species known to date. FAO Fish. Syn., Roma, 125(16): I-VIII, 382p.

IHERING, R. V. (1940), 1968, Dicionário dos Animais do Brasil. Editora Universidade de Brasília, 790p.
MACHADO, L. et alii, 1997, Alimentação natural de "garoupas", Epinephelus marginatus (Perciformes-Serranidae) na região de Barra do Sul-SC. VII Congresso Latinoamericano sobre Ciências do Mar - Resumos Expandidos, Santos, II: 114-115.

MENEZES, N. A. \& FIGUEIREDO, J. L., 1985, Manual de peixes marinhos do sudeste do Brasil. V. Teleostei (4). Universidade de São Paulo/Museu de Zoologia, São Paulo, 105p.

MENNI, R. C., 1983, Los peces en el medio marino. Estudio Sigma S. R. L., Buenos Aires, 181p.

MOYLE, P. B. \& CECH Jr., J. J., 1982, Fishes: An Introduction to Ichthyology. Prentice-Hall, Inc., Englewood Cliffs, $607 \mathrm{p}$.

NAGELKERKEN, W. P., 1981, Distribution and ecology of the groupers (Serranidae) and snappers (Lutjanidae) of the Netherlands Antilles. Foundation for Scientific Research in Surinan and the Netherlands/Natural History Series, Utrech, 3: 1-71.

NOMURA, H., 1984, Dicionário dos Peixes do Brasil. Editerra Editorial Ltda, Brasília, 482p.

PAIVA, M. P., 1997, Recursos pesqueiros estuarinos e marinhos do Brasil. Edições UFC, Fortaleza, 286p.

PAIVA, M. P. \& ANDRADE, M. F., 1994, Pescarias de barcos linheiros ao largo da costa sudeste do Brasil (19791985). B. Tec. Inst. Pesca, São Paulo, 18: 1-21.

PAIVA, M. P. \& ANDRADE-TUBINO, M. F., 1998, Produção, esforço e produtividade da pesca de linheiros ao largo da costa sudeste do Brasil (1979-1995). UFRJ Bol. Rec. Pesq., Rio de Janeiro, 1: 1-21.

PAIVA, M. P. \& FONTELES-FILHO, A. A., 1995, Distribuição e abundância de alguns peixes bentônicos na área de Abrolhos (Brasil). Arq. Ciên. Mar., Fortaleza, 29(1/2): 36-41.

SANTOS, E. (1949), 1982, Nossos Peixes Marinhos (Vida e costume dos peixes do Brasil). Editora Itatiaia Limitada, Belo Horizonte, 265p.

SILVA, P. C. M., 1973, A ressurgência em Cabo Frio. Publ. Inst. Pesq. mar., Rio de Janeiro, 78: 1-56.

SUZUKI, C. R., (1983) 1993, Guia de peixes do litoral brasileiro. Edições Marítimas Ltda., Rio de Janeiro, 404p.

SZPILMAN, M., 1991, Guia Aqualung de Peixes. Guia prático de identificação dos peixes do litoral brasileiro. Aqualung Confecção Ltda., Rio de Janeiro, 284p.

VALENTIN, J. L., 1984, Analyse des paramètres hydrobiologiques dans la remontée de Cabo Frio (Brésil). Marine Biology, Berlin, 82: 259-276.

VAZZOLER, A. E. A. M., 1993, Ecologia de peixes marinhos do Brasil. Encontro Brasileiro de Ictiologia, São Paulo, 10: 264-286. 